EUROPEAN ORGANIZATION FOR NUCLEAR RESEARCH

CERN - AB Department

CERN-AB-2007-051

\title{
LONGITUDINAL COUPLED-BUNCH INSTABILITIES IN THE CERN PS
}

\author{
H. Damerau, S. Hancock, M. Mehler ${ }^{1)}$, C. Rossi, E. Shaposhnikova, J. \\ Tückmantel, J.-L. Vallet
}

CERN, Geneva, Switzerland

\begin{abstract}
Longitudinal coupled bunch instabilities in the CERN PS represent a major limitation to the high brightness beam delivered for the LHC. To identify possible impedance sources for these instabilities, machine development studies have been carried out. The growth rates of coupled bunch modes have been measured, and modes have been identified using mountain range data. Growth rate estimations from coupled bunch mode theory are compared to these results. It is shown that the longitudinal impedance of the broad resonance curve of the main $10 \mathrm{MHz}$ RF system can be identified as the most probable source. Several modes are driven simultaneously due to the large width of the resonance, which is considered for the analysis.
\end{abstract}

\footnotetext{
${ }^{1)}$ GSI, Darmstadt, Germany
}

Presented at

PAC07, 22nd PAC Conference, June 25-29, 2007, Albuquerque, USA

Geneva, Switzerland

August 2007 


\title{
LONGITUDINAL COUPLED-BUNCH INSTABILITIES IN THE CERN PS
}

\author{
H. Damerau*, S. Hancock, C. Rossi, E. Shaposhnikova, J. Tuckmantel, J.-L. Vallet, \\ CERN, Geneva, Switzerland \\ M. Mehler, GSI, Darmstadt, Germany
}

\begin{abstract}
Longitudinal coupled bunch instabilities in the CERN PS represent a major limitation to the high brightness beam delivered for the LHC. To identify possible impedance sources for these instabilities, machine development studies have been carried out. The growth rates of coupled bunch modes have been measured, and modes have been identified using mountain range data. Growth rate estimations from coupled bunch mode theory are compared to these results. It is shown that the longitudinal impedance of the broad resonance curve of the main $10 \mathrm{MHz}$ RF system can be identified as the most probable source. Several modes are driven simultaneously due to the large width of the resonance, which is considered for the analysis.
\end{abstract}

\section{INTRODUCTION}

Longitudinal coupled bunch (CB) oscillations, especially coupled dipole oscillations of bunches around their reference position, represent a major limitation to the beam intensity and brightness that can be delivered from the CERN PS. To damp or suppress the development of CB instabilities effectively, their excitation mechanism and the source of impedance exciting the beam need to be understood. Machine development (MD) studies have been performed during the machine run in 2006 with a high brightness proton beam, comparable to the brightness of the LHC-type beams in the PS.

The CERN PS is equipped with ten $2.8-10 \mathrm{MHz}$ tunable ferrite-loaded cavities for acceleration, as well as 20,40 , 80 and $200 \mathrm{MHz}$ cavities for RF manipulations and longitudinal emittance blow-up. To simplify the mode identification, seven bunches (full ring) have been injected and accelerated at $h=7(3.05$ to $3.34 \mathrm{MHz})$. The 20,40 and $80 \mathrm{MHz}$ RF systems were switched off with their gaps short circuited. The CB instabilities start shortly after transition crossing $\left(\gamma_{\operatorname{tr}}=6.1\right)$ and develop during acceleration. Firstly, growth rates were measured by analyzing the beam signal around a specific harmonic of the revolution frequency and secondly, the spectrum of the CB modes has been extracted from mountain range measurements.

\section{COUPLED-BUNCH OSCILLATIONS}

The indication of $\mathrm{CB}$ oscillations are spectral lines of the beam at

$$
\omega=(n+l M) \omega_{0}+m \omega_{s}
$$

\footnotetext{
*heiko.damerau@cern.ch
}

where $l=0, \pm 1, \ldots \pm \infty, \omega_{\mathrm{RF}}=h \omega_{0}$ is the RF frequency at harmonic $h, \omega_{s}$ is the synchrotron frequency and $\omega_{0}$ is the revolution frequency.

A single bunch can perform dipole $(m=1)$, quadrupole $(m=2)$ and higher order $(m=3 \ldots \infty)$ oscillations of the bunch distribution. Additionally, the oscillation between two bunches may be coupled: all $M$ bunches of a batch oscillate with the same single bunch oscillation type $m$ and amplitude $\hat{\phi}$, but with a constant phase advance of $\Delta \phi=$ $2 \pi n / M$ between bunches; the CB mode number is given by $n=0 \ldots M-1$ [1].

A mixture of different $\mathrm{CB}$ oscillation modes $m, n$ is generally observed. Selection of a specific mode of interest can be done either by measuring the beam signal within a limited bandwidth only, or by decomposition using a discrete Fourier transform (DFT).

\section{GROWTH RATE MEASUREMENTS}

Growth rates of longitudinal CB oscillations can be measured by observing the frequency spectrum of the beam signal from a longitudinal pick-up around the harmonics of the revolution frequency. Parts of the narrow-band CB damping loops for LHC type beams have been used for observation (Fig. 1). The beam signal from a longitudinal pick-up

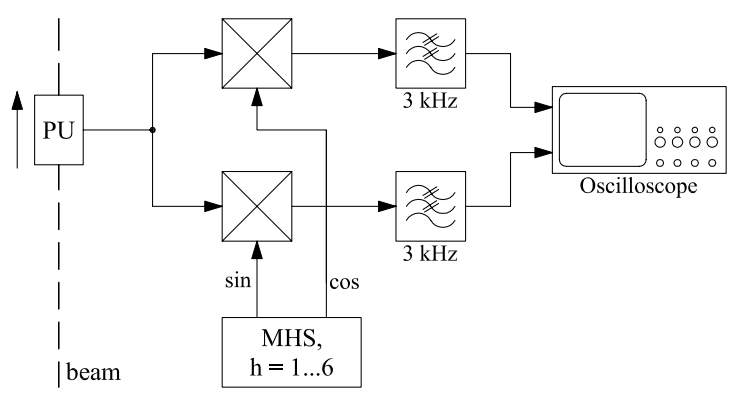

Figure 1: Set-up for the growth rate measurements. The multi-harmonic source (MHS) is a DDS oscillator generating a selectable harmonic of the revolution frequency.

is mixed with a selectable revolution harmonic generated by a DDS oscillator coupled to the revolution frequency of the beam. The base-band $I / Q$ signals are then filtered by a low-pass filter cutting above $3 \mathrm{kHz}$ and recorded with an oscilloscope. A typical measurement is shown in Fig. 2. Transition crossing is clearly visible as a peak of the peak detected beam signal. Thereafter, the base-band signals grow exponentially, indicating a CB instability. 


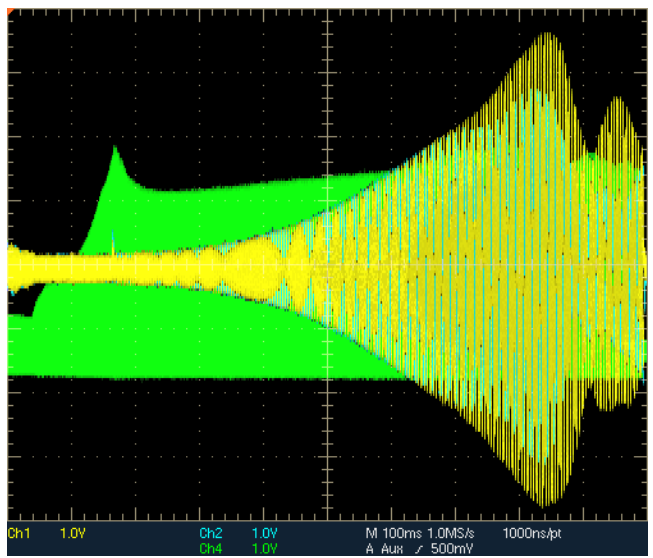

Figure 2: Growth rate measurements of a CB mode ( $n=2$, $5, m=1,2, \ldots) ; f_{s} \simeq 0.2 \mathrm{kHz}$ after transition. Green trace: peak detected beam signal; yellow and blue traces: down-converted base-band signals around the 5 th harmonic of $\omega_{0}$; horizontal scale: $100 \mathrm{~ms}$ per division.

The growth rate $1 / \tau$ of the $\mathrm{CB}$ oscillation is computed from a linear fit to the logarithmic amplitude envelope of the measured signal in the region of exponential growth; saturation is thus not taken into account. The measured growth rates versus longitudinal bunch emittance are plotted in Fig. 3. The emittance is adjusted with a controlled longitudinal blow-up at flat-bottom. Only a very weak de-
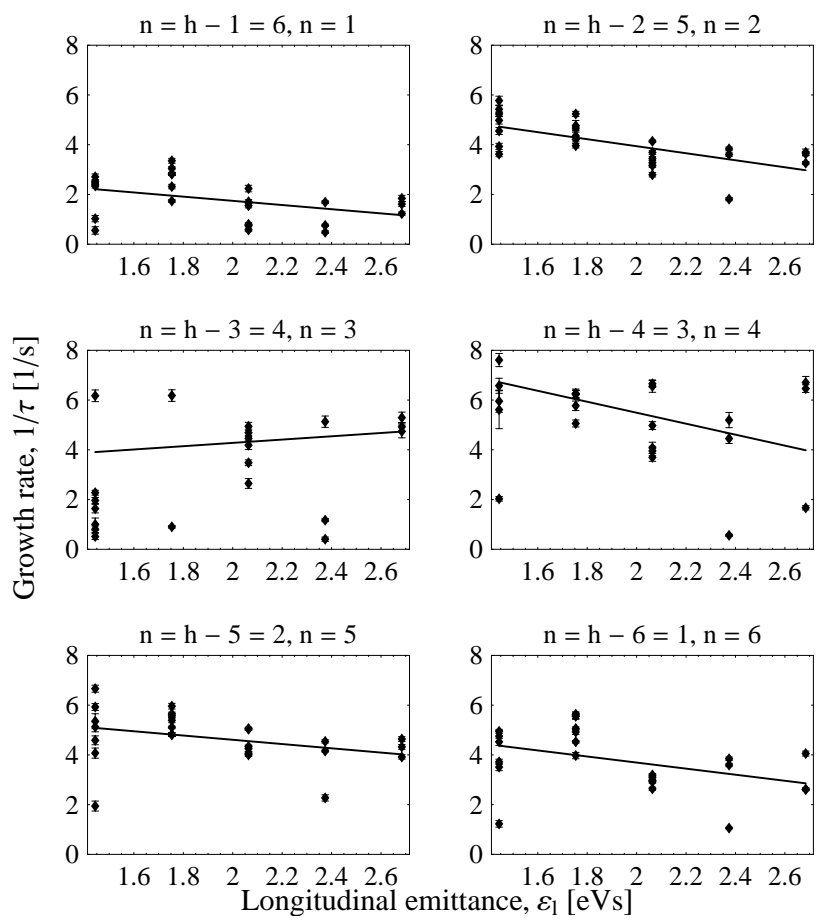

Figure 3: Growth rates $1 / \tau$ versus longitudinal emittance $\varepsilon_{l}$, measured at the harmonics 1 to 6 of $\omega_{0}$.

pendence of the growth rates on the longitudinal bunch emittance is observed. This indicates an impedance source in the area with flat dependence of the growth rate on bunch length (for $m=1: \tau_{\text {bunch }} \simeq 0.5 / f_{r}$ ). The large scatter of the measurements can be related to small intensity variations of each individual cycle. The $n=3$ and 4 modes start to develop $200 \mathrm{~ms}$ later in the cycle and have larger scatter since the bunches already oscillate on the other modes.

\section{MODE ANALYSIS}

The dipole mode CB spectrum at a certain moment in the cycle can be found from mountain range measurements spanning a few periods of the synchrotron oscillation (Fig. 4). In this case, the center positions of the bunches
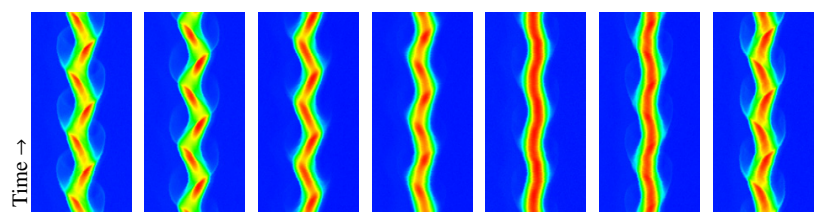

Figure 4: Mountain range density plots (490 ms after transition, see Fig. 2) of all seven bunches during $15 \mathrm{~ms}\left(f_{s} \simeq\right.$ $210 \mathrm{~Hz}$ ). The horizontal span of each frame is $160 \mathrm{~ns}$; the distance between two bunches (reduced) is $304 \mathrm{~ns}$.

versus time is computed from the center position of a Gaussian fit to each bunch in each frame. A nearly sinusoidal motion of the bunch centers with time is observed. A fit of a sinusoidal function to the motion of the bunch centers

$$
\hat{\phi}_{k} \sin \left(\omega_{s} t+\theta_{k}\right)=\sum_{n=0}^{M-1} \hat{\Phi}_{n} \sin \left(\omega_{s} t+\frac{2 \pi n k}{M}+\Theta_{n}\right)
$$

results in an oscillation amplitude $\hat{\phi}_{k}(k$ : bunch index), an oscillation phase $\theta_{k}$ and a frequency, corresponding to $f_{s}$. The $n=0 \ldots 6$ mode amplitudes $\hat{\Phi}_{n}$ and phases $\Theta_{n}$ are computed applying a DFT according to

$$
\hat{\Phi}_{n} e^{-i \Theta_{n}}=\frac{1}{M} \sum_{k=0}^{M-1} \hat{\phi}_{k} e^{-i \theta_{k}} \cdot e^{2 \pi i k n / M} .
$$

Fig. 5 illustrates the CB mode spectrum obtained from the mountain range data shown above. This mode spectrum

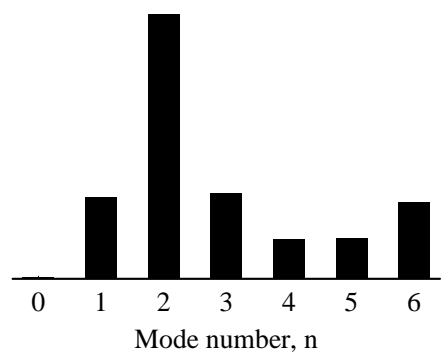

Figure 5: Dipole CB mode amplitudes $\hat{\Phi}_{n}$ extracted from the mountain range measurement shown in Fig. 4.

is very reproducible. The $n=2$ oscillation is excited first and has the maximum amplitude. 


\section{SOURCES OF IMPEDANCE}

Obvious sources of longitudinal impedance in the PS are the RF cavities. Only the 10 and $200 \mathrm{MHz}$ RF systems were not short-circuited. The frequency of the $200 \mathrm{MHz}$ RF system is too high to explain the dependence on bunch length and the excitation of dipole modes. Therefore, the $10 \mathrm{MHz}$ RF system [2] is the most probable impedance source. A simplified model of the system is sketched in Fig. 6.

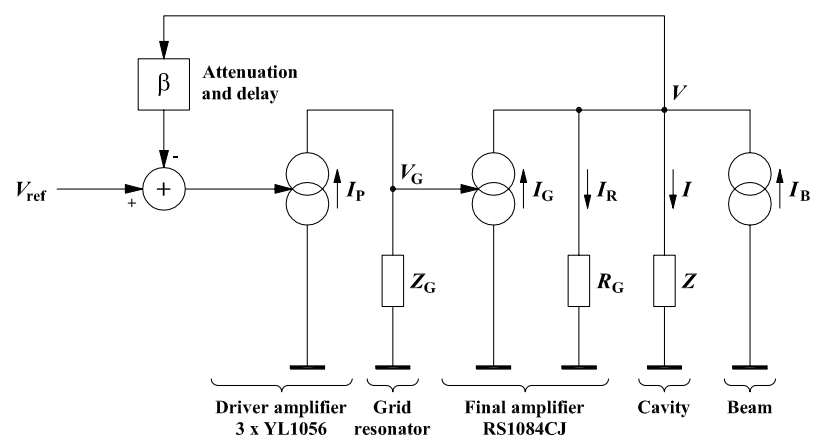

Figure 6: Simplified model of the $10 \mathrm{MHz}$ RF system, including ferrite loaded cavities in the PS and power amplifier with a tuned grid resonator and the fast feedback around the amplifier.

According to this model, the impedance seen by the beam can be written as

$$
Z_{\mathrm{c}}=\frac{d V}{d I_{B}}=\frac{Z_{G} g_{P} g_{G}}{Z_{G} g_{P} g_{G} \beta+\left(R_{G}+Z\right) /\left(R_{G} Z\right)},
$$

where $Z_{G}(\omega)$ is the impedance of the grid resonator, $g_{P}$ and $g_{G}$ are the effective trans-conductances of driver and final amplifier, $\beta$ represents attenuation and delay of the feedback loop. The parameters of the model have been matched to reproduce the measured open and closed loop transfer functions of six of the ten cavities. The result for $\operatorname{Re}\left\{Z_{c}\right\}$ is shown in Fig. 7.

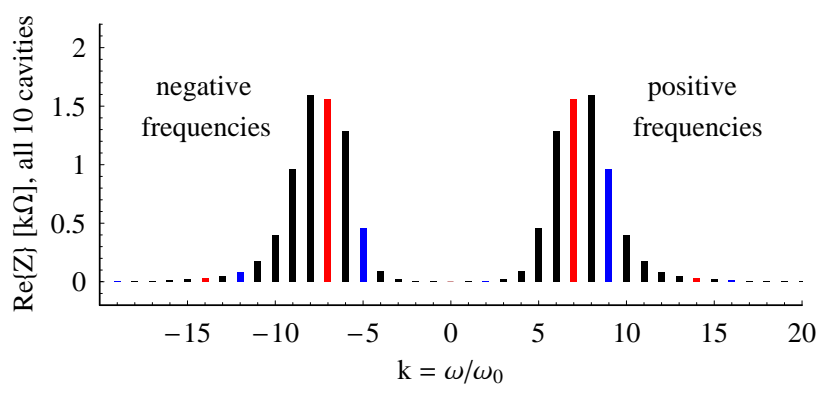

Figure 7: Real part of the total impedance of the $10 \mathrm{MHz}$ cavities at the revolution harmonics.

As the impedance $Z_{c}$ covers several revolution harmonics around $h \omega_{0}$, the narrow-band approximation is not applicable for the estimation of the growth rates of the $\mathrm{CB}$ oscillations. For dipole modes, the growth rates $1 / \tau=$
$\operatorname{Im}\left\{\left(\Omega-\omega_{s 0}\right) / \omega_{s 0}\right\}$ are given by the eigenvalues, $\lambda_{k}$ of the system $[3,4]$

$$
\lambda_{k} j_{k} \propto \sum_{l^{\prime}=-\infty}^{\infty} \frac{Z_{c}\left(k^{\prime}\right)}{k^{\prime}} j_{k^{\prime}} \int_{-\infty}^{\infty} \frac{d g(r)}{d r} J_{1}\left(k^{\prime} r\right) J_{1}(k r) d r .
$$

The synchrotron frequency in the center of the bunch is given by $\omega_{s 0}$ and $J_{1}$ is the Bessel function of the first order; $k=n+l M, k^{\prime}=n+l^{\prime} M$; the eigenvector (Fourier transform of the current perturbation) is $j_{k}$.

Solving the system Eq. (5) for its eigenvalues $\lambda_{k}$ results in the growth rates shown in Table 1. Due to the symme-

Table 1: CB growth rates from eigenvalues of Eq. (5).

\begin{tabular}{l|ccc|} 
Mode number & $n=1$ & $n=2$ & $n=3$ \\
Growth rate, $1 / \tau$ & $2.5 \mathrm{~s}^{-1}$ & $3.0 \mathrm{~s}^{-1}$ & $1.0 \mathrm{~s}^{-1}$ \\
\hline
\end{tabular}

try of Eq. (5), the modes $n=6,5,4$ have the same, but negative growth rates as modes $n=1,2,3$ (Table 1 ). The measured growth rates are slightly higher. A delayed occurrence of the $n=3$ mode, when other CB modes are already well developed, may explain the large scattering measured (compare Fig. 3).

\section{CONCLUSION}

Longitudinal $\mathrm{CB}$ growth rates and mode spectra measurements in the CERN PS indicate that the main $10 \mathrm{MHz}$ RF system represents the most probable impedance source for excitation. Due to the strong feedback, the impedance of the cavities covers 4 harmonics at each side of the RF harmonic. The shape of these impedance lines predicts a $n=2$ mode being stronger than $n=1$. This has also been observed with beam. Methods to damp the CB oscillations with a wide-band feedback or by optimizing the cavity impedance with the one-turn-delay feedback are currently studied.

Work supported by EU Design Study DIRACsecondaryBeams (contract 515873).

\section{REFERENCES}

[1] F. Pedersen, F. Sacherer, "Theory and Performance of the Longitudinal Active Damping System for the CERN PS Booster", PAC'77, IEEE Trans. Nucl. Sci., Vol. NS-28, 1977, pp. 1396-1398

[2] D. Grier, "The PS $10 \mathrm{MHz}$ Cavity and Power Amplifier", PS/RF Note 2002-073, CERN, Geneva, Switzerland, 2002

[3] J. L. Laclare, "Bunched Beam Coherent Instabilities", CERN 87-03, CAS on General Acc. Phys., Oxford, U. K., 1985, pp. 264-326

[4] E. Shaposhnikova, "Analysis of Coupled Bunch Instability Spectra”, CERN-SL-99-040 HRF, CERN, Geneva, Switzerland, 1999 